\title{
Interconnect Tuning Strategies for High-Performance ICs*
}

\author{
Andrew B. Kahng, Sudhakar Muddu, Egino Sarto and Rahul Sharma \\ Silicon Graphics, Inc., Mountain View, CA 94039 \\ \{muddu,sarto,ashu\}@mti.sgi.com,abk@cs.ucla.edu
}

\begin{abstract}
Interconnect tuning is an increasingly critical degree of freedom in the physical design of high-performance VLSI systems. By interconnect tuning, we refer to the selection of line thicknesses, widths and spacings in multi-layer interconnect to simultaneously optimize signal distribution, signal performance, signal integrity, and interconnect manufacturability and reliability. This is a key activity in most leading-edge design projects, but has received little attention in the literature. Our work provides the first technology-specific studies of interconnect tuning in the literature. We center on global wiring layers and interconnect tuning issues related to bus routing, repeater insertion, and choice of shielding/spacing rules for signal integrity and performance. We address four basic questions. (1) How should width and spacing be allocated to maximize performance for a given line pitch? (2) For a given line pitch, what criteria affect the optimal interval at which repeaters should be inserted into global interconnects? (3) Under what circumstances are shield wires the optimum technique for improving interconnect performance? (4) In global interconnect with repeaters, what other interconnect tuning is possible? Our study of question (4) demonstrates a new approach of offsetting repeater placements that can reduce worstcase cross-chip delays by over $30 \%$ in current technologies.
\end{abstract}

\section{Introduction}

With technology scaling, on-chip interconnect becomes an increasingly critical determinant of performance, manufacturability and reliability in high-end VLSI designs. Current and future designs are generally interconnect-limited, and the available routing resource must be carefully balanced among signal distribution, power/ground distribution, and clock distribution. Table 1 reproduces several technology projections from the 1997 SIA National Technology Roadmap for Semiconductors [1]. A notable deviation from the original 1994 Roadmap is that maximum onchip clock frequencies will reach the gigahertz range even in the $180 \mathrm{~nm}$ process generation. The implications of technology scaling - particularly for system interconnect - are very complicated. Example considerations for a 7-layer metal (7LM) process might include:

- Local interconnect layers (e.g., M1-M3) should generally remain at near-minimum dimensions and pitch in order to achieve routing density (for an example analysis of interconnect density in $0.25 \mu \mathrm{m}$ processes, see [10]). For short lines (e.g., several hundred microns or less), thinner metal offers less lateral coupling capacitance and driver loading, and thus locally improves circuit performance. At the same time, maximum wire width is limited by the aspect ratio upper bound. The resulting thin and narrow wires are highly resistive and also subject to reliability concerns; they are hence unsuitable for global interconnects, power distribution, etc. We also note that layers M2-M3 (and maybe M4) will support a mix of local and "near-global" wiring, e.g., long wires within a single block. The distribution of lengths and performance goals for these signals can vary considerably between designs; since shorter wires are better routed on thinner metal, these design-specific considerations will affect the interconnect.

${ }^{*}$ Principal author, to whom correspondence should be addressed: Dr. Sudhakar Muddu, Silicon Graphics, Inc., muddu@mti.sgi.com. Andrew B. Kahng is associate professor of computer science at UCLA.
- Power distribution layers (e.g., M6-M7, maybe M5), which typically also support the top-level clock distribution (mesh or balanced -tree), should be as thick as possible for reliability. IR drop and clock skew - as well as robustness under process variations - also suggest the use of thick wire on these layers. Thick wire additionally conserves area, but can suffer from increased lateral capacitive coupling.

- Global interconnect layers (e.g., M4-M6) support inter-block signal runs with length on the order of $3000 \mu \mathrm{m}-15000 \mu \mathrm{m}$. To satisfy delay and signal integrity constraints, at least three degrees of freedom are available: line width and spacing, repeater insertion, and shield wiring. Repeater insertion shields downstream capacitance and is the canonical means of converting "quadratic" $R C$ delay into "near-linear" delay; this technique also improves edge rates and hence noise immunity. When lateral coupling capacitances are large, worst-case "Miller coupling" begins to dominate noise and delay calculations; this is alleviated by increasing the line spacing and/or adding shield wiring (i.e., wires connected to ground), with future techniques possibly including dedicated ground and power planes interleaved with signal layers [5]. ${ }^{1}$ Another technique to reduce the lateral coupling capacitance is to interleave signal lines which do not switch at the same signal transistion period. The bus-dominated nature of global interconnects in building-block and high-performance designs only worsens the effects of coupling, since it results in longer parallel runs.

- All layers are subject to mutual pitch-matching, via sizing, etc. considerations. Hence, available widths and spacings on one layer are not independent of the widths and spacings on a second layer.

The above are only a few of the applicable design considerations; the net effect is that balancing interconnect resources is now extremely difficult as designs move into and beyond the quarter-micron regime.

\section{Interconnect Strategies}

Interconnect tuning is the selection by a design team of line thicknesses, widths and spacings in multi-layer interconnect to simultaneously achieve: (i) distribution (available wiring density) for local signals, global signals, clock, power and ground; (ii) performance (signal propagation delay), particularly on global interconnects; (iii) noise immunity (signal integrity), again particularly on global interconnects; and (iv) manufacturability and reliability (e.g., required margins for AC self-heat or DC electromigration on interconnects, short-circuit power in attached devices, etc.). Today, interconnect tuning is a key activity in most leadingedge microprocessor projects. It is clearly an option whenever the design and fabrication are owned by a single entity; however, for highvolume projects even fabless design houses are exercising increasing

\footnotetext{
${ }^{1}$ When two parallel neighboring lines $L 1$ and $L 2$ switch simultaneously in opposite directions, the driver of $L 1$ sees the grounded line capacitance plus twice the coupling capacitance of $L 1$ to $L 2$. If $L 2$ is quiet when $L 1$ switches, then the driver of $L 1$ sees the grounded line capacitance plus the coupling capacitance to $L 2$. And if $L 2$ switches simultaneously in the opposite direction, the driver of $L 1$ sees only the grounded line capacitance. (In leadingedge processes, each neighbor coupling is of the same (and possibly greater) magnitude as the area coupling to ground.) The "coupling factor" or "switching factor" is often given in the range $[0,2]$, and since most lines have two neighbors, the total coupling factor is in the range $[0,4]$.
} 


\begin{tabular}{|l|c|c|c|c|c|c|}
\hline SIA National Technology Roadmap (1997) \\
\hline Year & 1997 & 1999 & 2001 & 2003 & 2006 & 2009 \\
\hline Minimum feature size - dense lines $(\mathrm{nm})$ & 250 & 180 & 150 & 130 & 100 & 70 \\
\hline High-end on-chip clock frequency $(\mathrm{MHz})$ & 750 & 1250 & 1400 & 1600 & 2000 & 2500 \\
\hline \# Wiring layers & 6 & $6-7$ & 7 & 7 & $7-8$ & $8-9$ \\
\hline Minimum contacted M1 pitch $(\mu \mathrm{m})$ & 0.64 & 0.46 & 0.40 & 0.34 & 0.26 & 0.19 \\
\hline Metal height/width aspect ratio & $1.8: 1$ & $1.8: 1$ & $2.0: 1$ & $2.1: 1$ & $2.4: 1$ & $2.7: 1$ \\
\hline
\end{tabular}

Table 1: Selected technology projections from the 1997 SIA NTRS.

influence on vendors' processes [10]. Nevertheless, this topic has received very little attention in the literature, with only a small handful of high-level treatments available. ${ }^{2}$

Our work is the first in the literature to attempt a wide-ranging study of interconnect tuning. We center on global wiring layers (e.g., M4 and M5 in a 6LM process), and interconnect tuning issues related to bus routing, repeater insertion, and choice of shielding/spacing rules for signal integrity and performance. ${ }^{3}$ (Of necessity, our studies are for now independent of several other issues, e.g., wire tapering and choice of wire thickness.)

\begin{tabular}{|c|c|c|c|c|c|}
\hline \multirow{2}{*}{$\begin{array}{c}\text { Width,Space } \\
(\mu \mathrm{m})\end{array}$} & \multicolumn{5}{|c|}{ Coupling Capacitance per $\mu \mathrm{m}(\mathrm{aF})$} \\
\cline { 2 - 6 } & Left Neighbor & Right Neighbor & $\begin{array}{c}\text { Top } \\
\text { Plane }\end{array}$ & $\begin{array}{c}\text { Bottom Plane } \\
\text { (ground })\end{array}$ & Total \\
\hline $1.0,2.2$ & 25.20 & 25.61 & 54.79 & 46.84 & 152.66 \\
\hline $1.2,2.0$ & 29.00 & 29.26 & 56.74 & 48.22 & 163.53 \\
\hline $1.4,1.8$ & 33.33 & 33.11 & 57.76 & 51.53 & 177.32 \\
\hline $1.6,1.6$ & 38.71 & 38.60 & 59.09 & 51.90 & 188.41 \\
\hline $1.8,1.4$ & 44.75 & 44.12 & 60.22 & 51.52 & 200.92 \\
\hline
\end{tabular}

Table 2: Summary of M3 coupling capacitances extracted using QuickCap. Bottom M2 is a ground plane; top M4 is populated by crossover lines.

We address four basic questions.

1. How should width and spacing be allocated to maximize performance for a given line pitch?

2. For a given line pitch, what criteria affect the optimal interval at which repeaters should be inserted into global interconnects?

3. Under what circumstances are shield wires the optimum technique for improving interconnect performance?

4. In global interconnect with repeaters, what other interconnect tuning is possible?

We answer these questions using technology parameters from a representative 0.25 um CMOS process; this matches the process technology context for many current- and next-generation microprocessors. Coupling capacitance studies are performed with the commercial QuickCap 3-D field solver, and interconnect delay and noise coupling studies are performed with the commercial HSPICE simulator. Of particular interest is our study of question (4): we demonstrate that a new methodology for offsetting repeater placements can reduce worst-case cross-chip delays by over $30 \%$ in current technologies, versus traditional repeater insertion methodology.

${ }^{2}$ For example, [12] describes a characterization and analysis methodology and the need to break ideal scaling in deep submicron interconnect. [8] is another work that centers on analysis of a given multi-layer interconnect process, as opposed to the underlying interconnect tuning. [3] and [6] are examples of system-level treatments based on Rent's rule for interconnect length distribution.

${ }^{3}$ Even though the results presented in this paper are for aluminum interconnects with $\mathrm{SiO} 2$ dielectric, similar techniques can be applied for copper interconnects and low-K dielectrics.

\section{Allocation of Width and Spacing for Given Pitch}

Our first study seeks to determine how width and spacing should be optimally allocated for a given line pitch. In practice, the actual line width used is considerably greater than the minimum line width achievable in lithography. Thus, there is freedom to tune the width and spacing once assumptions are in place for line thickness and target line length. We note that because very long inter-block lines will have repeaters inserted regularly (see Section 3 below), the maximum line length of interest is equal to the optimum interval between repeaters; this length ranges between $2500 \mu \mathrm{m}$ and $5000 \mu \mathrm{m}$ for global interconnect layers in leadingedge technologies.

We have performed detailed studies of "fast" M3 interconnect with $3.2 \mu \mathrm{m}$ pitch, assuming that M2 crossunders are dense (i.e., can be approximated as a ground plane) [9] and explicitly modeling M4 crossovers Dielectric modeling is based on actual layer data for a representative $0.25 \mu \mathrm{m}$ CMOS process. QuickCap was used to extract coupling and area capacitances, summarized in Table 1. As is typical in such analyses, we assume worst-case coupling, i.e., a total coupling factor of 4.0 (worst-case coupling factor of 2.0 to each of the left and right neighbors of the (victim) line under analysis).

Table 3 shows HSPICE-computed line delays for M3 line lengths ranging from $4000 \mu \mathrm{m}$ to $6000 \mu \mathrm{m}$. Again, dense M2 is assumed to be a ground plane, and M4 crossovers are modeled explicitly. The Table shows that (width,spacing) $=(1.2,2.0) \mu \mathrm{m}$ gives the best performance for the given line pitch.

\section{Bounding the Interval Between Repeaters}

A very basic study (in some sense a pre-requisite to all other interconnect tuning) asks how often repeaters should be inserted into global interconnects. This is of course a chicken-egg problem, in that the optimum repeater interval depends on the interconnect tuning, and the interconnect tuning depends on the maximum run ever made without an intervening repeater. However, the following can be noted.

- A body of study shows that repeaters should be inserted at uniform intervals. In other words, there should be a constant interconnect length (or interconnect delay) between each pair of adjacent repeaters; the first and last segments of the path are exceptions because in practice the driver and receiver sizes may not be the same as the repeater size. Actually, such theoretical results deviate from real-life practice. On any source-destination path the repeater sizes need not be the same. It may also be better to add repeaters in parallel in order to drive larger wire lengths. (This is not just for performance: repeaters locally affect device area and routing constraints. However, our studies have not yet addressed such layout issues. Using the same principle (and with certain types of methodology and chip planning constraints), it can be better to increase the size of the drivers inside the block as much as possible, which would increase the first segment length.

Assuming that the driver size and the receiver size are the same as the size of the repeaters inserted along the path, we calculate the total delay, optimal number of repeaters and optimal distance 


\begin{tabular}{|c||c|c|c||c|c|c||c|c|c|}
\hline \multirow{2}{*}{$\begin{array}{c}\text { Width,Space } \\
(\mu \mathrm{m})\end{array}$} & \multicolumn{10}{||}{$50 \%$ Threshold Rise Delay $(\mathrm{ps})$} \\
\cline { 2 - 10 } & $\begin{array}{c}\text { Driver } \\
\text { Load Delay }\end{array}$ & $\begin{array}{c}\text { Int. } \\
\text { Delay }\end{array}$ & $\begin{array}{c}\text { Total } \\
\text { Delay }\end{array}$ & $\begin{array}{c}\text { Driver } \\
\text { Load Delay }\end{array}$ & $\begin{array}{c}\text { Int. } \\
\text { Delay }\end{array}$ & $\begin{array}{c}\text { Total } \\
\text { Delay }\end{array}$ & $\begin{array}{c}\text { Driver } \\
\text { Load Delay }\end{array}$ & $\begin{array}{c}\text { Int. } \\
\text { Delay }\end{array}$ & $\begin{array}{c}\text { Total } \\
\text { Delay }\end{array}$ \\
\hline $1.0,2.2$ & 106.19 & 113.99 & 220.17 & 132.74 & 168.36 & 301.10 & 159.28 & 233.09 & 392.37 \\
\hline $1.2,2.0$ & 115.00 & 100.72 & 215.73 & 143.76 & 149.26 & 293.02 & 172.51 & 207.14 & 379.65 \\
\hline $1.4,1.8$ & 126.61 & 92.80 & 219.41 & 158.27 & 138.04 & 296.31 & 189.92 & 192.10 & 382.02 \\
\hline $1.6,1.6$ & 138.77 & 87.12 & 225.89 & 173.46 & 130.04 & 303.04 & 208.15 & 181.41 & 389.56 \\
\hline $1.8,1.4$ & 151.24 & 82.84 & 234.08 & 189.04 & 124.03 & 313.08 & 226.85 & 173.41 & 400.26 \\
\hline
\end{tabular}

Table 3: Delay estimates for various M3 line configurations. Driver and receiver buffer sizes: $(\mathrm{wp}=100 \mu \mathrm{m}, \mathrm{wn}=50 \mu \mathrm{m})$. Delay is computed from input of driver to input of receiver.

\begin{tabular}{|c|c|c|r|r|r|r|r|}
\hline $\begin{array}{c}\text { Driver/Receiver } \\
(\mathrm{wp}, \mathrm{wn})(\mu \mathrm{m})\end{array}$ & $\begin{array}{c}\text { Width } \\
(\mu \mathrm{m})\end{array}$ & $\begin{array}{c}\text { Space } \\
(\mu \mathrm{m})\end{array}$ & $\begin{array}{r}\text { Length } \\
(\mu \mathrm{m})\end{array}$ & SF & $\begin{array}{r}\text { Delay } \\
(\mathrm{ps})\end{array}$ & $\begin{array}{r}\text { Rise Time } \\
(\mathrm{ps})\end{array}$ & $\begin{array}{r}\text { Fall Time } \\
(\mathrm{ps})\end{array}$ \\
\hline$(130,65) /(130,65)$ & 1 & 1.1 & 10000 & 4 & 589 & 1679 & 1510 \\
\hline$(130,65) /(130,65)$ & 1 & 1.1 & 9000 & 4 & 486 & 1421 & 1265 \\
\hline$(130,65) /(130,65)$ & 1 & 1.1 & 8000 & 4 & 393 & 1187 & 1044 \\
\hline$(130,65) /(130,65)$ & 1 & 1.1 & 7000 & 4 & 310 & 975 & 847 \\
\hline$(130,65) /(130,65)$ & 1 & 1.1 & 5000 & 4 & 172 & 623 & 525 \\
\hline$(130,65) /(130,65)$ & 1 & 1.1 & 10000 & 3 & 488 & 1405 & 1267 \\
\hline$(130,65) /(130,65)$ & 1 & 1.1 & 9000 & 3 & 404 & 1193 & 1066 \\
\hline$(130,65) /(130,65)$ & 1 & 1.1 & 8000 & 3 & 327 & 1001 & 885 \\
\hline$(130,65) /(130,65)$ & 1 & 1.1 & 7000 & 3 & 259 & 828 & 723 \\
\hline$(130,65) /(130,65)$ & 1 & 1.1 & 5000 & 3 & 147 & 538 & 458 \\
\hline$(130,65) /(130,65)$ & 1 & 1.1 & 10000 & 2 & 388 & 1131 & 1026 \\
\hline$(130,65) /(130,65)$ & 1 & 1.1 & 9000 & 2 & 323 & 966 & 869 \\
\hline$(130,65) /(130,65)$ & 1 & 1.1 & 8000 & 2 & 263 & 817 & 728 \\
\hline$(130,65) /(130,65)$ & 1 & 1.1 & 7000 & 2 & 209 & 682 & 601 \\
\hline$(130,65) /(130,65)$ & 1 & 1.1 & 5000 & 2 & 120 & 456 & 393 \\
\hline$(130,65) /(130,65)$ & 1.4 & 1.6 & 10000 & 4 & 366 & 1123 & 980 \\
\hline$(130,65) /(130,65)$ & 1.4 & 1.6 & 9000 & 4 & 303 & 963 & 832 \\
\hline$(130,65) /(130,65)$ & 1.4 & 1.6 & 8000 & 4 & 246 & 818 & 698 \\
\hline$(130,65) /(130,65)$ & 1.4 & 1.6 & 7000 & 4 & 195 & 686 & 578 \\
\hline$(130,65) /(130,65)$ & 1.4 & 1.6 & 5000 & 4 & 111 & 465 & 384 \\
\hline$(130,65) /(130,65)$ & 1.4 & 1.6 & 10000 & 3 & 320 & 992 & 869 \\
\hline$(130,65) /(130,65)$ & 1.4 & 1.6 & 9000 & 3 & 266 & 854 & 740 \\
\hline$(130,65) /(130,65)$ & 1.4 & 1.6 & 8000 & 3 & 217 & 729 & 625 \\
\hline$(130,65) /(130,65)$ & 1.4 & 1.6 & 7000 & 3 & 172 & 615 & 522 \\
\hline$(130,65) /(130,65)$ & 1.4 & 1.6 & 5000 & 3 & 99 & 422 & 352 \\
\hline$(130,65) /(130,65)$ & 1.4 & 1.6 & 10000 & 2 & 275 & 862 & 759 \\
\hline$(130,65) /(130,65)$ & 1.4 & 1.6 & 9000 & 2 & 229 & 746 & 650 \\
\hline$(130,65) /(130,65)$ & 1.4 & 1.6 & 8000 & 2 & 188 & 640 & 553 \\
\hline$(130,65) /(130,65)$ & 1.4 & 1.6 & 7000 & 2 & 150 & 543 & 465 \\
\hline$(130,65) /(130,65)$ & 1.4 & 1.6 & 5000 & 2 & 87 & 382 & 322 \\
\hline & & & & & & & \\
\hline
\end{tabular}

Table 4: Summary of M3 interconnect slew times. M4 is top layer; M1 is bottom layer. Two combinations of width/spacing are shown, along with three different coupling factor assumptions. The input slew time is $400 \mathrm{ps}$ and the output slew times are computed as $10 \%-90 \%$ for rise time and $90 \%-10 \%$ for fall time.

between the repeaters.

The total delay for a path with $K$ repeaters is

$$
T_{\text {tot }}^{K}=T_{\text {first_stage }}+(K-1) * T_{\text {Rep_stage }}+T_{\text {Final_stage }}
$$

The delay of the first stage is the total delay from the output of driver to the input of the first repeater, i.e., $T_{\text {first_stage }}=T_{g d}+T_{\text {int }}$, where gate load delay is $T_{g d}=R_{\text {rep }}\left(C_{\text {int }}^{e f f}+C_{r e p}\right)$, interconnect delay is $T_{i n t}=R_{\text {int }}\left(C_{i n t} / 2+C_{\text {rep }}\right)$, and $R_{\text {rep }}, C_{\text {rep }}$ are repeater output resistance and input gate capacitance. The effective capacitance at the gate output can be approximated as $C_{\text {int }}^{\text {eff }}=\alpha C_{i n t}$ where $\alpha$ is a constant between $1 / 6$ and 1 [11]. Let $L_{p}^{\text {int }}$ be the interconnect path length between driver and receiver. Then for op- timal placement of repeaters the interconnect length between repeaters is $\frac{L_{p}}{K+1}$. Therefore, the total delay for the path is

$$
\begin{aligned}
T_{\text {tot }}^{K}= & (K+1) *\left(T_{g d}+T_{\text {int }}\right) \\
= & (K+1) * R_{r e p}\left(\alpha * c * \frac{L_{p}}{K+1}+C_{r e p}\right) \\
& +r * L_{p}\left(c * \frac{L_{p}}{2(K+1)}+C_{r e p}\right)
\end{aligned}
$$

where $r, c$ are resistance and capacitance per unit length of the interconnect line. We compute the optimal number of repeaters that minimizes total delay by setting $\frac{\partial T_{t o t}}{\partial K}=0$, and obtain

$$
K=\sqrt{\frac{r c L_{p}^{2}}{2 R_{\text {rep }} C_{r e p}}}-1
$$

To minimize total delay, gate load delay and interconnect delay should be equal. If effective capacitance is not considered in the gate load delay computation, and with current technology trends, gate load delay will always be greater than interconnect delay. Under these conditions, to minimize total delay one can increase the time of flight (or wire length) between repeaters until slew time constraints become tight. In the current range of $0.35 \mu \mathrm{m}$ and $0.25 \mu \mathrm{m}$ process generations, global interconnects have repeaters inserted with periods ranging from $2500 \mu \mathrm{m}$ to $10000 \mu \mathrm{m}$.

- Repeater insertion is also driven by pure interconnect delay, since larger time of flight implies larger slew time on the transition seen at the receiver. Edges with large slew times cause much larger gate delays, are more susceptible to noise, are more susceptible to process-distribution influenced delay variations, and also increase the short-circuit power dissipation. Even in today's designs, slew times above 600-700 ps cannot be tolerated. Thus, even without the delay minimization objective, edge rate control will force insertion of repeaters.In fact, some of the functionality of "post-layout optimization" tools for gate sizing and repeater insertion is driven by edge rate checks as opposed to signal delay reduction.

- In practice, repeaters will be implemented using inverters whenever possible, due to performance and area efficiency.

Table 4 summarizes M3 interconnect slew times for line width $1.0 \mu \mathrm{m}$ and line spacing $1.2 \mu \mathrm{m}$ (corresponding to a "dense" M3 routing pitch), and input slew time of $400 \mathrm{ps}$. All capacitance extractions were performed with QuickCap, and correspond to M4 and M1 as the top and bottom ground planes, respectively. Switching factors range from 4 (both neighbors switching in the opposite direction from the victim) to 2 (both neighbors quiet, or one neighbor switching in the opposite direction and 


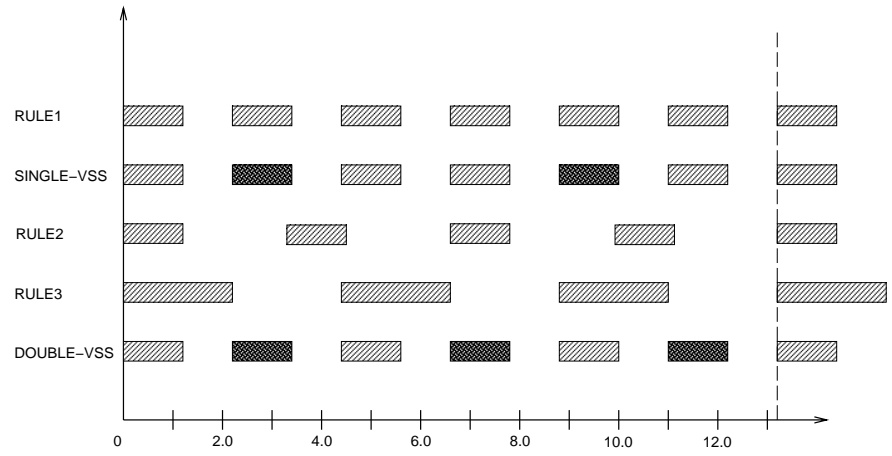

Figure 1: Pitch-matched width-spacing rules. Rule1 allows six lines per $13.2 \mu \mathrm{m}$; Rule 2 and the Single- $V_{S S}$ rule (Rule1 width/spacing, but every third line grounded) both allow four signal lines per $13.2 \mu \mathrm{m}$; and Rule 3 and the Double- $V_{S S}$ rule (Rule1 width/spacing, but every other line grounded) both allow three signal lines per $13.2 \mu \mathrm{m}$.

one neighbor switching in the same direction with respect to the victim). We see that the M3 distance between repeaters has an upper bound of $5000 \mu \mathrm{m}$ due to edge rate considerations alone. Separate studies show that this upper bound on distance between repeaters is essentially unaffected by changes to the driver/receiver sizing or the input slew time.

\section{Benefits of Shield Wiring}

Our third study addresses the question of whether shield wiring is an effective means of improving delay and signal integrity performance of long global interconnects. We consider various width-spacing rules for M3 interconnect, in order to evaluate the utility of spacing vs. shielding techniques. Our evaluations are with respect to delay only; for all of the configurations, the assumed slew time upper bounds of approximately 600ps imply that noise coupling will not be problematic. Figure 1 contrasts five pitch-matched width-spacing rules:

- Rule1: $1.2 \mu \mathrm{m}$ width, $1.0 \mu \mathrm{m}$ spacing

- Single- $V_{S S}: 1.2 \mu \mathrm{m}$ width, $1.0 \mu \mathrm{m}$ spacing, with every third line grounded (i.e., every signal line has one grounded neighbor to shield it)

- Rule2: $1.2 \mu \mathrm{m}$ width, $2.1 \mu \mathrm{m}$ spacing

- Rule3: $2.2 \mu \mathrm{m}$ width, $2.2 \mu \mathrm{m}$ spacing

- Double- $V_{S S}: 1.2 \mu \mathrm{m}$ width, $2.1 \mu \mathrm{m}$ spacing, with every other line grounded (i.e., every signal line has two grounded neighbors to shield it)

Again, QuickCap was used to extract capacitive couplings of a given victim line to its neighbor lines and the neighboring top/bottom layers; these results are shown in Table 5. Notice that the Rule1, Rule 2 and Rule3 rules have worst-case coupling factors $=4$. On the other hand, the Single- $V_{S S}$ rule has worst-case coupling factor $=3$, and the Double$V_{S S}$ rule has worst-case coupling factor $=2$. Table 6 shows the delay performance for a $4000 \mu \mathrm{m}$ M3 line, under various bottom ground and top plane configurations. We observe:

- The Rule3 rule provides $37 \%$ decrease in total delay, but since $C_{e f f}$ was not used in the gate load delay computation, actual delay reductions could be even greater.

- The Single- $V_{S S}$ rule is less effective than the Rule2 rule; note that the two rules are equivalent in terms of effective routing density. Our studies have not yet addressed the routing interactions that can potentially affect this analysis. In particular, shield lines may be added to bring power and ground connections to repeater blocks.
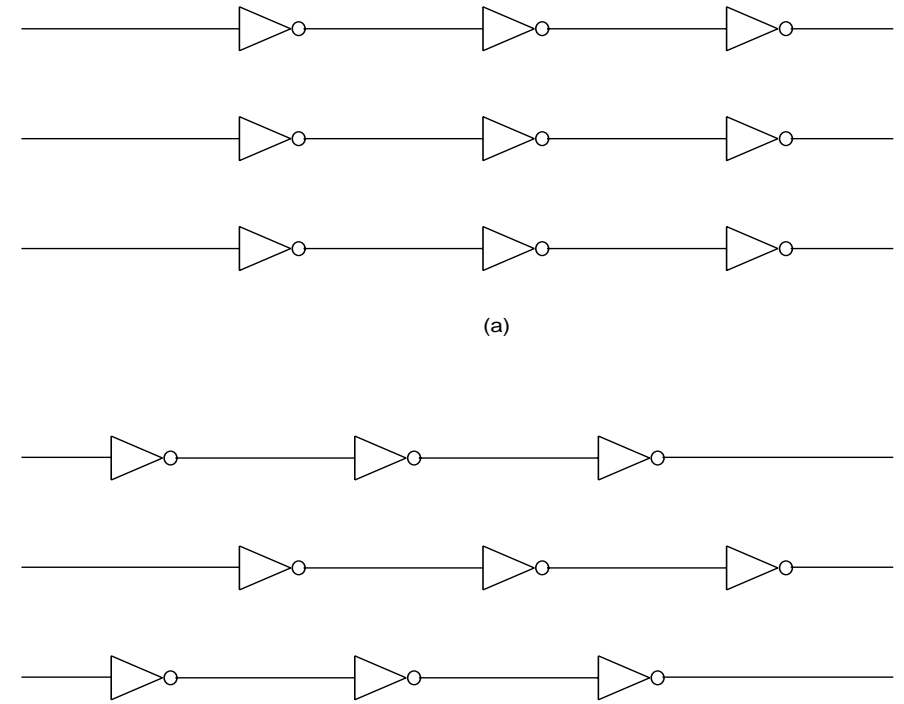

(b)

Figure 2: Reduction of worst-case Miller coupling by offsetting inverters. In (a), inverters on the left and right neighbor lines are at phase $=0$ with respect to the inverters on the middle line. In (b), inverters on the left and right neighbors are at phase $=0.5$.

- The Double- $V_{S S}$ rule gives improved total delays compared with the Rule 3 rule, with the rules being equivalent in terms of effective routing density. However, the Rule 3 rule yields smaller interconnect delays, so that driver size reductions have greater potential for delay improvement. Thus, the Rule 3 rule seems preferable. When two buses have activity patterns such that each is quiet when the other is active, then their lines can be interleaved such that they effectively follow the Double- $V_{S S}$ rule. In such a case, interleaving is clearly superior to the Rule 3 rule, since the effective routing density is doubled.

- Gate load delays are larger than interconnect delays, suggesting that it is preferable to decrease line widths and increase line spacings. We also note that a dense M4 top layer decreases total delay, and a dense M2 bottom (ground plane) layer decreases total delay for smaller line widths only.

\section{New Repeater Offset Methodology for Global Buses}

Finally, we study another form of tuning that is possible for global interconnects. Our motivations are three-fold: (i) global interconnect is increasingly dominated by wide buses; (ii) present methodology designs global interconnects for worst-case Miller coupling; and (iii) present methodology routes long global buses using repeater blocks, i.e., blocks of co-located inverters spaced every, say, $4000 \mu \mathrm{m}$.

We have proposed a simple method to improve global interconnect performance. The idea is to reduce the worst-case Miller coupling by offsetting the inverters on adjacent lines (see Figure 2). In the previous methodology (Figure 2(a)), the worst-case switching of a neighbor line (i.e., simultaneously and in the opposite direction to the switching of the victim line) persists through the entire chain of inverters. However, with offset inverter locations (Figure 2(b)), any worst-case simultaneous switching on a neighbor line persists only for half of each period between consecutive inverters, and furthermore becomes best-case simultaneous switching for the other half of the period!.

To confirm the advantages of this method, the following experimental methodology was used. 


\begin{tabular}{|c|c|c|c|c|c|c|c|}
\hline \multirow[b]{2}{*}{ M3 Rules } & \multirow[b]{2}{*}{$\begin{array}{c}\text { Width,Space } \\
(\mu \mathrm{m})\end{array}$} & \multirow[b]{2}{*}{$\begin{array}{l}\text { Ground,Top } \\
\text { Planes }\end{array}$} & \multicolumn{5}{|c|}{ Coupling Capacitance per $\mu \mathrm{m}(\mathrm{aF})$} \\
\hline & & & Left Neighbor & Right Neighbor & $\begin{array}{c}\text { Top } \\
\text { Plane }\end{array}$ & $\begin{array}{l}\text { Bottom Plane } \\
\text { (ground) }\end{array}$ & Total \\
\hline Rule1 & $1.2,1.0$ & Substrate,M4 Line & 68.23 & 68.15 & 43.68 & 14.79 & 195.03 \\
\hline Rule1 & $1.2,1.0$ & M2,M4 Line & 60.30 & 60.92 & 43.96 & 34.88 & 202.37 \\
\hline Rule1 & $1.2,1.0$ & $\mathrm{M} 2,-$ & 74.67 & 74.23 & - & 42.99 & 192.44 \\
\hline Rule2 & $1.2,2.1$ & Substrate,M4 Line & 36.87 & 34.37 & 58.58 & 18.07 & 148.29 \\
\hline Rule2 & $1.2,2.1$ & M2,M4 Line & 26.96 & 27.10 & 58.51 & 48.72 & 160.41 \\
\hline Rule2 & $1.2,2.1$ & $\mathrm{M} 2,-$ & 42.17 & 42.43 & - & 59.15 & 143.96 \\
\hline Rule3 & $2.2,2.2$ & Substrate,M4 Line & 35.09 & 36.50 & 77.61 & 22.14 & 171.52 \\
\hline Rule3 & $2.2,2.2$ & M2,M4 Line & 26.18 & 25.61 & 77.51 & 67.92 & 198.82 \\
\hline Rule3 & $2.2,2.2$ & M2,-- & 44.33 & 43.86 & - & 73.23 & 162.14 \\
\hline
\end{tabular}

Table 5: M3 coupling capacitances extracted using QuickCap for various interconnect tuning rules and combinations of bottom and top planes.

\begin{tabular}{|c|c|c|c|c|c|c|}
\hline \multirow[b]{2}{*}{ M3 Rules } & \multirow[b]{2}{*}{$\begin{array}{l}\text { Width,Space } \\
(\mu \mathrm{m})\end{array}$} & \multirow[b]{2}{*}{$\begin{array}{l}\text { Ground,Top } \\
\text { Planes }\end{array}$} & \multicolumn{3}{|c|}{$50 \%$ threshold rise delay $(\mathrm{ps})$} & \multirow{2}{*}{$\begin{array}{l}\text { \% Gain } \\
\text { w.r.t. } \\
\text { Rule1 }\end{array}$} \\
\hline & & & $\begin{array}{c}\text { Driver Load } \\
\text { Delay }\end{array}$ & $\begin{array}{c}\text { Interconnect } \\
\text { Delay }\end{array}$ & $\begin{array}{l}\text { Total } \\
\text { Delay }\end{array}$ & \\
\hline Rule1 & $1.2,1.0$ & Substrate,M4 Line & 173.04 & 116.88 & 289.92 & - \\
\hline Rule1 & $1.2,1.0$ & M2,M4 Line & 167.84 & 114.03 & 281.87 & - \\
\hline Rule1 & $1.2,1.0$ & $\mathrm{M} 2,-$ & 178.03 & 119.62 & 297.65 & - \\
\hline Rule2 & $1.2,2.1$ & Substrate,M4 Line & 114.47 & 84.75 & 199.22 & 29 \\
\hline Rule2 & $1.2,2.1$ & M2,M4 Line & 112.50 & 83.66 & 196.16 & 30 \\
\hline Rule1 with Single VSS & $1.2,1.0$ & Substrate,M4 Line & 137.41 & 97.34 & 234.75 & 17 \\
\hline Rule1 with Single VSS & $1.2,1.0$ & M2,M4 Line & 136.17 & 96.66 & 232.83 & 17 \\
\hline Rule1 with Single VSS & $1.2,1.0$ & M2,-- & 139.14 & 98.28 & 237.42 & 16 \\
\hline Rule2 & $1.2,2.1$ & $\mathrm{M} 2,-$ & 119.29 & 87.39 & 206.68 & 27 \\
\hline Rule3 & $2.2,2.2$ & Substrate,M4 Line & 126.91 & 49.95 & 176.85 & 37 \\
\hline Rule3 & $2.2,2.2$ & M2,M4 Line & 130.08 & 50.90 & 180.98 & 36 \\
\hline Rule3 & $2.2,2.2$ & $\mathrm{M} 2,-$ & 130.40 & 50.99 & 181.39 & 36 \\
\hline Rule1 with Double VSS & $1.2,1.0$ & Substrate,M4 Line & 99.74 & 78.11 & 177.85 & 37 \\
\hline Rule1 with Double VSS & $1.2,1.0$ & M2,M4 Line & 104.34 & 80.83 & 185.17 & 34 \\
\hline Rule1 with Double VSS & $1.2,1.0$ & $\overline{\mathrm{M} 2,-}$ & 121.14 & 78.53 & 199.67 & 29 \\
\hline
\end{tabular}

Table 6: Delay estimates for a $4000 \mu \mathrm{m}$ M3 line, under various interconnect tuning configurations. Driver and receiver buffer sizes: $(w p=100 \mu \mathrm{m}, \mathrm{wn}=50 \mu \mathrm{m})$. Delay is computed from input of driver to input of receiver.

- We study systems of three parallel interconnect lines, with lengths either $10000 \mu \mathrm{m}$ or $14000 \mu \mathrm{m}$. These lines are stimulated by a waveform with risetime $=$ falltime $=200 \mathrm{ps}$. The middle line is considered the "victim" for analysis purposes.

- We model two "technologies" representative of M3 and M4 in an $0.25 \mu \mathrm{m}$ CMOS process. In each technology, line resistance is $50 \Omega$ per $1000 \mu \mathrm{m}$. In Technology I, capacitive couplings to left neighbor, ground and right neighbor per $1000 \mu \mathrm{m}$ are respectively $60 \mathrm{fF}, 80 \mathrm{fF}$ and $60 \mathrm{fF}$. In Technology II, capacitive couplings to left neighbor, ground and right neighbor per $1000 \mu \mathrm{m}$ are respectively 80fF, $160 \mathrm{fF}$ and $80 \mathrm{fF}$.

- We assume a period between inverters (repeaters) of $4000 \mu \mathrm{m}$. So that HSPICE cannot introduce any error in its RC analysis, we manually distributed the line and coupling parasitics into $40 \mu \mathrm{m}$ segments, i.e., repeaters occurred every 100 segments, and line lengths were 250 or 350 segments. Each segment is modeled as a double-pi model. ${ }^{4}$

- We always place the inverters on the middle line with "phase = $0 "$, i.e., at positions $4000,8000, \ldots$ microns along the line. Inverters on the left and right neighbors are placed according to all combinations of phase $=0,0.1,0.2, \ldots, 0.9$ (again with respect to

\footnotetext{
${ }^{4}$ This segmenting is chosen such that any finer-grain representation does not change the HSPICE-computed delays.
}

the period of $4000 \mu \mathrm{m})$. There are 100 different phase combinations. Figure 2 shows the three-line configurations with left/right neighbor phase combinations of $(0,0)$ and $(0.5,0.5)$.

- We stimulate the three lines with the periodic waveform, with the first transition either rising $(\mathrm{R})$ or falling $(\mathrm{F})$. There are eight combinations of directions for the first transisions, i.e., RRR, RRF, ... FFF.

- Finally, we may offset the input waveforms of the left and right neighbors by $-100 \mathrm{ps}, 0 \mathrm{ps}$ or $+100 \mathrm{ps}$ with respect to the input waveform of the middle line. There are nine combinations of these input offsets.

Table 7 shows HSPICE delays for systems of three lines of length $10000 \mu \mathrm{m}$, using Technology I, for all combinations of rising (R) and falling (F) initial transition on the input waveform. The Table shows delays for inverter phases $(0,0)$ and $(0.5,0.5)$ on the left and right neighbors of the middle line (phase 0 ). The effect of Miller coupling is clearly shown.

Table 8 shows the worst-case delays (with respect to all eight possible combinations of rising and falling inputs) for the middle line, for each combination of phases for the inverter locations on the left and right neighbor lines. Input offsets are all 0 , i.e., the waveforms start at the same time. All four combinations of Technology and line length are shown. In every case, the optimum phase combination is $(0.5,0.5)$, while the traditional phase combination of $(0.0,0.0)$ is actually the worst 


\begin{tabular}{|c|c|c|c|c|c|c|}
\hline \multirow{3}{*}{$\begin{array}{l}\text { Input waveforms } \\
\text { (Left neighbor, } \\
\text { victim, } \\
\text { right neighbor) }\end{array}$} & \multicolumn{6}{|c|}{ Interconnect Delay $(n s)$} \\
\hline & \multicolumn{3}{|c|}{ Left,right neighbor buffer phases: 0,0} & \multicolumn{3}{|c|}{ Left,right neighbor buffer phases:0.5,0.5 } \\
\hline & $\begin{array}{l}\text { Left neighbor } \\
\text { Delay }\end{array}$ & $\begin{array}{l}\text { Victim } \\
\text { Delay }\end{array}$ & $\begin{array}{l}\text { Right neighbor } \\
\text { Delay }\end{array}$ & $\begin{array}{l}\text { Left neighbor } \\
\text { Delay }\end{array}$ & $\begin{array}{l}\text { Victim } \\
\text { Delay }\end{array}$ & $\begin{array}{l}\text { Right neighbor } \\
\text { Delay }\end{array}$ \\
\hline $\mathrm{R}, \mathrm{R}, \mathrm{R}$ & 0.361 & 0.361 & 0.361 & 0.510 & 0.630 & 0.510 \\
\hline $\mathrm{R}, \mathrm{R}, \mathrm{F}$ & 0.428 & 0.584 & 0.676 & 0.533 & 0.697 & 0.499 \\
\hline $\mathrm{R}, \mathrm{F}, \mathrm{R}$ & 0.546 & 0.994 & 0.546 & 0.483 & 0.689 & 0.483 \\
\hline $\mathrm{R}, \mathrm{F}, \mathrm{F}$ & 0.676 & 0.584 & 0.428 & 0.499 & 0.697 & 0.533 \\
\hline $\mathrm{F}, \mathrm{R}, \mathrm{R}$ & 0.676 & 0.584 & 0.428 & 0.499 & 0.697 & 0.533 \\
\hline $\bar{F}, \mathrm{R}, \mathrm{F}$ & 0.546 & 0.994 & 0.546 & 0.483 & 0.689 & 0.483 \\
\hline $\mathrm{F}, \mathrm{F}, \mathrm{R}$ & 0.428 & 0.584 & 0.676 & 0.533 & 0.697 & 0.499 \\
\hline $\mathrm{F}, \mathrm{F}, \mathrm{F}$ & 0.361 & 0.361 & 0.361 & 0.510 & 0.630 & 0.510 \\
\hline
\end{tabular}

Table 7: HSPICE delays (ns) for three lines of length $10000 \mu m$, using Technology I, for all combinations of rising $(\mathrm{R})$ and falling $(\mathrm{F})$ initial transition on the input waveform. We show delays for inverter phases $(0,0)$ and $(0.5,0.5)$ on the left and right neighbors of the middle line (phase 0 ).

possible. The worst-case delay is reduced by anywhere from $25 \%$ to $30 \%$ when the repeaters are placed with optimum phase. Finally, Table 9 shows the same worst-case delays for the middle line, this time taken over all eight rise/fall combinations and all nine combinations of input waveform offsets. Again, even when the inputs do not switch perfectly simultaneously, the best phase combination is $(0.5,0.5)$ and the worst phase combination is the traditional $(0.0,0.0)$ methodology.

\section{Conclusions}

To our knowledge, this work has provided the first technology-specific studies of interconnect tuning in the literature. We have described experimental approaches to interconnect tuning issues related to bus routing, repeater insertion, and choice of shielding/spacing rules for signal integrity and performance. In particular, four questions have been addressed: allocation of width and spacing to maximize performance for a given pitch, finding the optimal interval for repeater insertion, assessing the potential benefits of shield wiring, and optimizing the insertion of repeaters in global buses. Our answers to these questions are at times surprising: in answering (3), we demonstrate that current shielding methodologies may be suboptimal when compared with alternate width/spacing rules, and in answering (4), we propose a new repeater offset technique that can reduce worst-case cross-chip delays by over $30 \%$ in current technologies. Ongoing efforts extend our interconnect tuning research to encompass layer thicknesses, more detailed analyses of noise coupling and tuning to meet noise margins, and the delay/noise behavior in emerging technology regimes ( $\mathrm{Cu}$ interconnect and low-K dielectrics). Finally, we seek to develop more complete full-chip interconnect tuning approaches based on analyses of the interconnect structure, speed target, and power dissipation target for a given design.

\section{REFERENCES}

[1] Semiconductor Industry Association, National Technology Roadmap for Semiconductors, December 1997.

[2] A. Deutsch, G. V. Kopcsay, C. W. Surovic, B. J. Rubin, L. M. Terman, R. P. Dunne and T. Gallo, "Modeling and Characterization of Long On-chip Interconnections for High-Performance Microprocessors", final report, ARPA HSCD Contract C-556003, September 1995. Also appeared in IBM Journal of Research and Development 39(5), Sept. 1995, pp. 547-567.

[3] P. D. Fisher, "Clock Cycle Estimations for Future Microprocessor Generations", Proc. IEEE Innovative Systems in Silicon, Austin, October 1997.

[4] L. Gwennap, "IC Makers Confront RC Limitations", Microdesign Resources Microprocessor Report, August 4, 1997, pp. 14-18.
[5] D. P. LaPotin, U. Ghoshal, E. Chiprout and S. R. Nassif, "Physical Design Challenges for Performance", International Symposium on Physical Design, April 1997, pp. 225-226.

[6] J. Meindl, "GigaScale Integration: 'Is the Sky the Limit'?", keynote presentation slides, Hot Chips IX, Stanford, CA, August 25-26, 1997.

[7] L. Scheffer, "A Roadmap of CAD Tool Changes for Sub-micron Interconnect Problems", International Symposium on Physical Design, April 1997, pp. 104-109.

[8] R. F. Sechler, "Interconnect design with VLSI CMOS", IBM Journal of Research and Development, Jan.-March 1995, pp. 23-31.

[9] J. Cong, L. He, A. B. Kahng, D. Noice, N. Shirali and S. H.-C. Yen, "Analysis and Justification of a Simple, Practical 2 1/2-D Capacitance Extraction Methodology", Proc. Design Automation Conference, June 1997.

[10] L. Gwennap, "IC Vendors Prepare for 0.25-Micron Leap", Microprocessor Report, September 16, 1996, pp. 11-15.

[11] A. B. Kahng and S. Muddu, "Efficient gate Delay Modeling for Large Interconnect Loads", Proc. IEEE Multi-Chip Module Conf., Feb. 1996.

[12] S.-Y. Oh, K.-J. Chang, N. Chang and K. Lee, 'Interconnect modeling and design in high-speed VLSI/ULSI systems", Proc. International Conference on Computer Design: VLSI in Computers and Processors, October 1992, pp. 184-189. 


\begin{tabular}{|c|c|c|c|c|c|c|c|c|c|c|c|}
\hline \multicolumn{12}{|c|}{ A. Line length $10000 \mu \mathrm{m}$, Technology I } \\
\hline & & \multicolumn{10}{|c|}{ Right Neighbor Phase } \\
\hline & & 0 & 0.1 & 0.2 & 0.3 & 0.4 & 0.5 & 0.6 & 0.7 & 0.8 & 0.9 \\
\hline \multirow{4}{*}{ Left } & 0 & 0.994 & 0.988 & 0.971 & $\begin{array}{ll}0.954 \\
\end{array}$ & 0.929 & 0.910 & 0.874 & 0.900 & 0.930 & 0.962 \\
\hline & \begin{tabular}{|l|}
0.1 \\
\end{tabular} & 0.988 & 0.974 & 0.960 & 0.938 & 0.911 & 0.885 & 0.854 & 0.881 & 0.917 & 0.952 \\
\hline & \begin{tabular}{|l|}
0.2 \\
\end{tabular} & 0.971 & 0.960 & 0.941 & 0.917 & 0.887 & 0.848 & 0.829 & 0.863 & 0.897 & 0.932 \\
\hline & \begin{tabular}{|l|l|}
0.3 \\
\end{tabular} & 0.954 & 0.938 & 0.917 & 0.890 & 0.855 & 0.806 & 0.801 & 0.834 & 0.872 & 0.912 \\
\hline \multirow[t]{2}{*}{ Neighbor } & \begin{tabular}{|l|l|}
0.4 \\
\end{tabular} & 0.929 & 0.911 & 0.887 & 0.855 & 0.818 & 0.753 & 0.766 & 0.805 & 0.841 & 0.885 \\
\hline & 0.5 & 0.910 & 0.885 & 0.848 & 0.806 & 0.753 & 0.697 & 0.735 & 0.778 & 0.822 & 0.867 \\
\hline \multirow{4}{*}{ Phase } & \begin{tabular}{|l|}
0.6 \\
\end{tabular} & 0.874 & 0.854 & 0.829 & 0.801 & 0.766 & 0.735 & 0.739 & 0.768 & 0.799 & 0.832 \\
\hline & \begin{tabular}{|l|}
0.7 \\
\end{tabular} & 0.900 & 0.881 & 0.863 & 0.834 & 0.805 & 0.778 & 0.768 & 0.796 & 0.827 & 0.859 \\
\hline & \begin{tabular}{|l|l|}
0.8 & \\
\end{tabular} & 0.930 & 0.917 & 0.897 & 0.872 & 0.841 & 0.822 & 0.799 & 0.827 & 0.860 & 0.894 \\
\hline & \begin{tabular}{|l|}
0.9 \\
\end{tabular} & 0.962 & 0.952 & 0.932 & 0.912 & 0.885 & 0.867 & 0.832 & 0.859 & 0.894 & 0.924 \\
\hline
\end{tabular}

\begin{tabular}{|c|c|c|c|c|c|c|c|c|c|c|c|}
\hline \multicolumn{12}{|c|}{ B. Line length $10000 \mu \mathrm{m}$, Technology II } \\
\hline & & & & & & sht Nei & abor Pha & & & & \\
\hline & & 0 & 0.1 & 0.2 & 0.3 & 0.4 & 0.5 & 0.6 & 0.7 & 0.8 & 0.9 \\
\hline \multirow{4}{*}{ Left } & 0 & $\mathbf{1 . 4 3 7}$ & 1.422 & 1.400 & 1.370 & 1.332 & 1.299 & 1.259 & 1.300 & 1.343 & 1.388 \\
\hline & 0.1 & 1.422 & 1.405 & 1.379 & 1.347 & 1.306 & 1.258 & 1.234 & 1.278 & 1.324 & 1.372 \\
\hline & 0.2 & 1.400 & 1.379 & 1.352 & 1.315 & 1.270 & 1.206 & 1.199 & 1.247 & 1.296 & 1.347 \\
\hline & 0.3 & 1.370 & 1.347 & 1.315 & 1.274 & 1.223 & 1.144 & 1.158 & $\frac{1.247}{1.208}$ & 1.261 & 1.314 \\
\hline \multirow[t]{2}{*}{ Neighbor } & \begin{tabular}{|l|}
0.5 \\
0.4
\end{tabular} & 1.332 & $\frac{1.341}{1.306}$ & $\begin{array}{l}1.515 \\
1.270\end{array}$ & $\frac{1.223}{1.223}$ & $\begin{array}{l}1.225 \\
1.167\end{array}$ & 1.1 .075 & $\begin{array}{l}1.150 \\
1.109\end{array}$ & $\frac{1.160}{1.161}$ & $\frac{1.201}{1.216}$ & $\begin{array}{l}1.514 \\
1.273\end{array}$ \\
\hline & 0.5 & 1.299 & 1.258 & 1.206 & 1.144 & 1.075 & 1.015 & 1.069 & 1.124 & 1.180 & 1.239 \\
\hline \multirow[t]{4}{*}{ Phase } & 0.6 & 1.259 & 1.234 & 1.199 & 1.158 & 1.109 & 1.069 & 1.079 & 1.120 & 1.163 & 1.209 \\
\hline & $\begin{array}{l}0.7 \\
\end{array}$ & 1.300 & 1.278 & 1.247 & 1.208 & 1.161 & 1.124 & 1.120 & 1.160 & 1.203 & 1.250 \\
\hline & 0.8 & 1.343 & 1.324 & 1.296 & 1.261 & 1.216 & 1.180 & 1.163 & 1.203 & 1.246 & 1.293 \\
\hline & $\begin{array}{l}0.9 \\
0.9\end{array}$ & $\frac{1.388}{1.388}$ & 1.372 & $\frac{1.257}{1.347}$ & $\frac{1.2014}{1.314}$ & 1.273 & 1.239 & 1.209 & $\frac{1.205}{1.250}$ & 1.293 & $\frac{1.239}{1.339}$ \\
\hline
\end{tabular}

\begin{tabular}{|c|c|c|c|c|c|c|c|c|c|c|c|}
\hline \multicolumn{12}{|c|}{ C. Line length $14000 \mu \mathrm{m}$, Technology I } \\
\hline & & \multicolumn{10}{|c|}{ Right Neighbor Phase } \\
\hline & & 0 & 0.1 & 0.2 & 0 & 0.4 & 0.5 & 0.6 & 0.7 & 0.8 & 0.9 \\
\hline \multirow{4}{*}{ Left } & 0 & 1.474 & 1.467 & 1.448 & 1.42 & 1.401 & 1.383 & 1.341 & 1.340 & 1.382 & 1.427 \\
\hline & 0.1 & 1.467 & 1.454 & 1.439 & 1.41 & 1.385 & 1.356 & 1.308 & 1.324 & 1.370 & 1.417 \\
\hline & 0.2 & 1.448 & 1.439 & 1.418 & 1.39 & 1.359 & 1.320 & 1.267 & 1.299 & 1.345 & 1.395 \\
\hline & 0.3 & 1.429 & 1.414 & 1.393 & 1.36 & 1.328 & 1.276 & 1.217 & 1.267 & 1.319 & 1.375 \\
\hline \multirow[t]{2}{*}{ Neighbor } & 0.4 & 1.401 & 1.385 & 1.359 & 1.32 & 1.287 & 1.223 & 1.174 & 1.229 & 1.285 & 1.342 \\
\hline & 0.5 & 1.383 & 1.356 & 1.320 & 1.27 & 1.223 & 1.105 & 1.146 & 1.203 & 1.263 & 1.323 \\
\hline \multirow[t]{4}{*}{ Phase } & 0.6 & 1.341 & 1.308 & 1.267 & 1.21 & 1.174 & 1.146 & 1.110 & 1.162 & 1.220 & 1.281 \\
\hline & 0.7 & 1.340 & 1.324 & 1.299 & 1.26 & 1.229 & 1.203 & 1.162 & 1.192 & 1.240 & 1.287 \\
\hline & 0.8 & 1.382 & 1.370 & 1.345 & 1.31 & 1.285 & 1.263 & 1.220 & 1.240 & 1.283 & 1.330 \\
\hline & 0.9 & 1.427 & 1.417 & 1.395 & 1.37 & 1.342 & 1.323 & 1.281 & 1.287 & 1.330 & 1.377 \\
\hline
\end{tabular}

\begin{tabular}{|c|c|c|c|c|c|c|c|c|c|c|c|}
\hline \multicolumn{12}{|c|}{ D. Line length $14000 \mu \mathrm{m}$, Technology II } \\
\hline & & \multicolumn{10}{|c|}{ Right Neighbor Phase } \\
\hline & & 0 & 0.1 & 0.2 & $\overline{0.3}$ & 0.4 & 0.5 & 0.6 & 0.7 & 0.8 & 0.9 \\
\hline \multirow{4}{*}{ Left } & 0 & 2.123 & 2.108 & 2.085 & 2.052 & 2.011 & 1.983 & 1.925 & 1.938 & 1.995 & 2.056 \\
\hline & \begin{tabular}{|l|}
0.1 \\
\end{tabular} & 2.108 & 2.092 & 2.064 & 2.029 & 1.985 & 1.943 & 1.876 & 1.913 & 1.974 & 2.039 \\
\hline & \begin{tabular}{|l|}
0.2 \\
\end{tabular} & 2.085 & 2.064 & 2.036 & 1.996 & 1.947 & 1.889 & 1.816 & 1.878 & 1.944 & 2.012 \\
\hline & 0.3 & 2.052 & 2.029 & 1.996 & 1.952 & 1.898 & 1.823 & 1.765 & 1.833 & 1.903 & 1.977 \\
\hline \multirow[t]{2}{*}{ Neighbor } & 0.4 & 2.011 & 1.985 & 1.947 & 1.898 & 1.837 & 1.743 & 1.703 & 1.778 & 1.854 & 1.932 \\
\hline & 0.5 & 1.983 & 1.943 & 1.889 & 1.82 & 1.743 & 1.590 & 1.664 & 1.744 & 1.823 & 1.903 \\
\hline \multirow[t]{4}{*}{ Phase } & 0.6 & 1.925 & 1.876 & 1.816 & 1.765 & 1.703 & 1.664 & 1.630 & 1.686 & 1.763 & 1.843 \\
\hline & 0.7 & 1.938 & 1.913 & 1.878 & 1.83 & 1.778 & 1.744 & 1.686 & 1.741 & 1.801 & 1.867 \\
\hline & 0.8 & 1.995 & 1.974 & 1.944 & 1.903 & 1.854 & 1.823 & 1.763 & 1.801 & 1.860 & 1.925 \\
\hline & 0.9 & 2.056 & 2.039 & 2.012 & 1.977 & 1.932 & 1.903 & 1.843 & 1.867 & 1.925 & 1.989 \\
\hline
\end{tabular}

Table 8: Worst-case middle line delays over all input rise/fall combinations, for each phase combination on left and right neighbors. Input offsets are all 0ps. 


\begin{tabular}{|c|c|c|c|c|c|c|c|c|c|c|c|}
\hline \multicolumn{12}{|c|}{ A. Line length $10000 \mu \mathrm{m}$, Technology I } \\
\hline & & \multicolumn{10}{|c|}{ Right Neighbor Phase } \\
\hline & & 0 & 0.1 & 0.2 & 0.3 & 0.4 & 0.5 & 0.6 & 0.7 & 0.8 & 0.9 \\
\hline \multirow{4}{*}{ Left } & 0 & $\mathbf{1 . 0 9 0}$ & 1.071 & 1.051 & 1.021 & 0.988 & 0.942 & 0.948 & 0.984 & 1.018 & 1.051 \\
\hline & 0.1 & 1.071 & 1.054 & 1.026 & 0.995 & 0.957 & 0.905 & 0.920 & 0.958 & 0.997 & 1.035 \\
\hline & 0.2 & 1.051 & 1.026 & 0.998 & 0.964 & 0.921 & 0.865 & 0.890 & 0.930 & 0.970 & 1.008 \\
\hline & 0.3 & 1.021 & 0.995 & 0.964 & 0.924 & 0.876 & 0.825 & 0.854 & 0.894 & 0.936 & 0.980 \\
\hline \multirow[t]{2}{*}{ Neighbor } & 0.4 & 0.988 & 0.957 & 0.921 & 0.876 & 0.825 & 0.782 & 0.813 & 0.856 & 0.900 & 0.944 \\
\hline & 0.5 & 0.942 & 0.905 & 0.865 & 0.825 & 0.782 & 0.760 & 0.791 & 0.824 & 0.860 & 0.900 \\
\hline \multirow[t]{4}{*}{ Phase } & 0.6 & 0.948 & 0.920 & 0.890 & 0.854 & 0.813 & 0.791 & 0.816 & 0.849 & 0.879 & 0.911 \\
\hline & 0.7 & 0.984 & 0.958 & 0.930 & 0.894 & 0.856 & 0.824 & 0.849 & 0.880 & 0.911 & 0.945 \\
\hline & 0.8 & 1.018 & 0.997 & 0.970 & 0.936 & 0.900 & 0.860 & 0.879 & 0.911 & 0.944 & 0.982 \\
\hline & 0.9 & 1.051 & 1.035 & 1.008 & 0.980 & 0.944 & 0.900 & 0.911 & 0.945 & 0.982 & 1.01 \\
\hline
\end{tabular}

\begin{tabular}{|c|c|c|c|c|c|c|c|c|c|c|c|}
\hline \multicolumn{12}{|c|}{ B. Line length $10000 \mu \mathrm{m}$, Technology II } \\
\hline & & & & & & sht Nei & abor Pha & & & & \\
\hline & & 0 & 0.1 & 0.2 & 0.3 & 0.4 & 0.5 & 0.6 & 0.7 & 0.8 & 0.9 \\
\hline \multirow{4}{*}{ Left } & 0 & $\mathbf{1 . 5 2 6}$ & 1.502 & 1.471 & 1.430 & 1.382 & 1.335 & 1.329 & 1.379 & 1.427 & 1.476 \\
\hline & 0.1 & 1.502 & 1.475 & 1.440 & 1.396 & 1.343 & 1.284 & 1.292 & 1.345 & 1.398 & 1.449 \\
\hline & 0.2 & 1.471 & 1.440 & 1.400 & 1.350 & 1.291 & 1.229 & 1.249 & 1.305 & 1.361 & 1.416 \\
\hline & 0.3 & 1.430 & 1.396 & 1.350 & 1.295 & 1.231 & 1.171 & 1.200 & 1.258 & 1.315 & 1.373 \\
\hline \multirow[t]{2}{*}{ Neighbor } & 0.4 & 1.382 & 1.343 & 1.291 & 1.231 & 1.167 & 1.114 & 1.148 & 1.205 & 1.262 & 1.321 \\
\hline & 0.5 & 1.335 & 1.284 & 1.229 & 1.171 & 1.114 & 1.074 & 1.124 & 1.175 & 1.226 & 1.279 \\
\hline \multirow[t]{4}{*}{ Phase } & 0.6 & 1.329 & 1.292 & 1.249 & 1.200 & 1.148 & 1.124 & 1.148 & 1.190 & 1.234 & 1.281 \\
\hline & 0.7 & 1.379 & 1.345 & 1.305 & 1.258 & 1.205 & 1.175 & 1.190 & 1.234 & 1.280 & 1.328 \\
\hline & 0.8 & 1.427 & 1.398 & 1.361 & 1.315 & 1.262 & 1.226 & 1.234 & 1.280 & 1.327 & 1.376 \\
\hline & 0.9 & 1.476 & 1.449 & 1.416 & 1.373 & 1.321 & 1.279 & 1.281 & 1.328 & 1.376 & 1.425 \\
\hline
\end{tabular}

\begin{tabular}{|c|c|c|c|c|c|c|c|c|c|c|c|}
\hline \multicolumn{12}{|c|}{ C. Line length $14000 \mu \mathrm{m}$, Technology I } \\
\hline & & \multicolumn{10}{|c|}{ Right Neighbor Phase } \\
\hline & & 0 & 0.1 & 0.2 & 0 . & 0.4 & 0.5 & 0.6 & 0.7 & 0.8 & 0.9 \\
\hline \multirow{4}{*}{ Left } & 0 & 1.572 & 1.551 & 1.530 & 1.50 & 1.465 & 1.419 & 1.391 & 1.429 & 1.474 & 1.521 \\
\hline & 0.1 & 1.551 & 1.534 & 1.507 & 1.47 & 1.438 & 1.388 & 1.362 & 1.406 & 1.451 & 1.499 \\
\hline & 0.2 & 1.530 & 1.507 & 1.474 & 1.44 & 1.400 & 1.345 & 1.323 & 1.373 & 1.423 & 1.475 \\
\hline & 0.3 & 1.502 & 1.472 & 1.442 & 1.40 & 1.353 & 1.293 & 1.279 & 1.334 & 1.388 & 1.443 \\
\hline \multirow[t]{2}{*}{ Neighbor } & 0.4 & 1.465 & 1.438 & 1.400 & 1.35 & 1.297 & 1.241 & 1.231 & 1.288 & 1.348 & 1.406 \\
\hline & 0.5 & 1.419 & 1.388 & 1.345 & 1.29 & 1.241 & 1.171 & 1.203 & 1.256 & 1.310 & 1.365 \\
\hline \multirow[t]{4}{*}{ Phase } & 0.6 & 1.391 & 1.362 & 1.323 & 1.27 & 1.231 & 1.203 & 1.206 & 1.247 & 1.291 & 1.339 \\
\hline & 0.7 & 1.429 & 1.406 & 1.373 & 1.33 & 1.288 & 1.256 & 1.247 & 1.288 & 1.332 & 1.377 \\
\hline & 0.8 & 1.474 & 1.451 & 1.423 & 1.38 & 1.348 & 1.310 & 1.291 & 1.332 & 1.374 & 1.424 \\
\hline & 0.9 & 1.521 & 1.499 & 1.475 & 1.44 & 1.406 & 1.365 & 1.339 & 1.377 & 1.424 & 1.471 \\
\hline
\end{tabular}

\begin{tabular}{|c|c|c|c|c|c|c|c|c|c|c|c|}
\hline \multicolumn{12}{|c|}{ D. Line length $14000 \mu \mathrm{m}$, Technology II } \\
\hline & & \multicolumn{10}{|c|}{ Right Neighbor Phase } \\
\hline & & 0 & 0.1 & 0.2 & 0.3 & 0.4 & 0.5 & 0.6 & 0.7 & 0.8 & 0.9 \\
\hline \multirow{4}{*}{ Left } & 0 & 2.213 & 2.190 & 2.157 & 2.116 & 2.069 & 2.031 & 1.974 & 2.027 & 2.084 & 2.147 \\
\hline & 0.1 & 2.190 & 2.161 & 2.125 & 2.081 & 2.029 & 1.982 & 1.930 & 1.991 & 2.053 & 2.119 \\
\hline & 0.2 & 2.157 & 2.125 & 2.085 & 2.035 & 1.977 & 1.920 & 1.879 & 1.946 & 2.013 & 2.084 \\
\hline & 0.3 & 2.116 & 2.081 & 2.035 & 1.980 & 1.913 & 1.846 & 1.818 & 1.893 & 1.965 & 2.041 \\
\hline \multirow[t]{2}{*}{ Neighbor } & 0.4 & 2.069 & 2.029 & 1.977 & 1.913 & 1.837 & 1.775 & 1.750 & 1.831 & 1.909 & $\begin{array}{l}1.989 \\
\end{array}$ \\
\hline & 0.5 & 2.031 & 1.982 & 1.920 & 1.846 & 1.775 & 1.666 & 1.730 & 1.804 & 1.879 & 1.955 \\
\hline \multirow[t]{4}{*}{ Phase } & 0.6 & 1.974 & 1.930 & 1.879 & 1.818 & 1.750 & 1.730 & 1.713 & 1.773 & 1.835 & 1.901 \\
\hline & 0.7 & 2.027 & 1.991 & 1.946 & 1.893 & 1.831 & 1.804 & 1.773 & 1.830 & 1.892 & 1.957 \\
\hline & 0.8 & 2.084 & 2.053 & 2.013 & 1.965 & 1.909 & 1.879 & 1.835 & 1.892 & 1.951 & 2.015 \\
\hline & 0.9 & 2.147 & 2.119 & 2.084 & 2.041 & 1.989 & 1.955 & 1.901 & 1.957 & 2.015 & 2.079 \\
\hline
\end{tabular}

Table 9: Worst-case delays with all combinations of input offsets. 